\title{
Utility of predictive equations for estimating the prevalence and incidence of primary open angle glaucoma in the UK
}

\author{
D C Minassian, A Reidy, M Coffey, A Minassian
}

\begin{abstract}
Aims-To assess the Tuck-Crick and the Quigley-Vitale predictive regression equations against fresh independent real world data for estimating the prevalence of primary open angle glaucoma (POAG) in the UK. To apply the equations to the elderly population of England and Wales, for which there is sample survey data on glaucoma, and demographic data.

Methods-Directly measured actual prevalence of POAG in a population sample of 1530 people was compared with the predicted prevalence derived by applying the Tuck-Crick and the Quigley-Vitale equations to the sample data. The two equations were applied to the demographic data of the population to project POAG prevalence and to derive 5 year cumulative incidence for the elderly population. These were compared with estimates derived from the local survey data. Results-The actual directly measured prevalence of POAG in the local sample was $3.01 \%$. The Tuck-Crick estimate was $2.74 \%$ (difference $0.27 \%$ ), and the Quigley-Vitale was $3.50 \%$ (difference $-0.49 \%)$. The three methods-projection from local survey data, Tuck-Crick, and Quigley-Vitale-gave point estimates of $2.85 \% \quad$ (228 526 cases), $2.71 \% \quad$ (217 375 cases), and $3.50 \%$ (280 364 cases) respectively for the prevalence of POAG in the elderly population of England and Wales (8 008705 people aged 65 or older). Calculation of incidence from age specific prevalence gave the following results: the numbers of new cases of POAG expected (5 year cumulative incidence) in the elderly population were 71146 and 94485 for methods 2 and 3 respectively.

Conclusions-The Tuck-Crick predictive equation performed well when applied to fresh (independent) actual data from a local sample survey using a particular definition for POAG, and its usefulness for estimation of prevalence of POAG in England and Wales has been demonstrated. The work on development of predictive equations has been very promising and further refinements could be made when more fresh survey data become available. (Br F Ophthalmol 2000;84:1159-1161)
\end{abstract}

D C Minassian, Institute of Ophthalmology, UCL, Bath Street, London EC1V 9EL UK

d.minassian@ucl.ac.uk

Accepted for publication 28 April 2000

The work by Quigley and Vitale in methods of predicting glaucoma prevalence in large populations ${ }^{1}$ has been followed by more recent work by Tuck and Crick. ${ }^{2}$ Both involved regression modelling-fitting of regression curves to published data from a large number of previous population surveys to arrive at a predictive regression equation that could be used to estimate glaucoma prevalence for any age (within the range of the survey data sets). To assess the accuracy of these potentially very useful equations (that is, how close the prediction might be to the actual prevalence), they need to be applied to and tested in fresh population samples, in which the actual prevalence of primary open angle glaucoma (POAG) has been directly measured.

We compare the actual prevalence of POAG in a sample of 1530 elderly people in the North London Eye Study (NLES), ${ }^{3}$ with the prevalence predicted by each of the two regression equations for the same sample. The NLES sample data were not available or not used when the regression equations were developed and are thus regarded as new suitable data for a "validation" of the two regression equations, in the context of the situation in the elderly population of England and Wales. Following this, we applied the two equations to the elderly population of England and Wales (excluding the small African-Caribbean ethnic group) to estimate the population prevalence of POAG, and compared the two estimates with that projected from the NLES age specific prevalence data.

\section{Methods}

PERFORMANCE OF THE PREDICTIVE EQUATIONS The North London Eye Study ${ }^{3}$ provided new data, not used in the development of the predictive equations. These comprised a random sample of 1547 people aged 65 and older, drawn from a defined population registered with 17 general practice groups. The sample represented a fairly average (not extremes) of socioeconomic mix and health status, as indicated by the Jarman scores, ${ }^{4}$ which ranged from 36.06 to -0.88 across the wards of the geographic area. For this analysis, 17/1547 belonging to African-Caribbean ethnic groups were excluded, leaving a sample of 1530 .

In the NLES, people were classified as "definite glaucoma cases" when there was an absolute field defect and, either a cup/disc ratio of 0.7 or larger or substantial asymmetry of the cups - that is, a difference in cup/disc ratio of 0.3 or larger between the two eyes. ${ }^{3}$

Each of the two predictive equations was 
the predicted number of POAG cases for the total sample of 1530 . The prevalence of POAG for each age (i), starting at 65 , was calculated by substituting the age in the predictive equations, as shown below:

The Tuck-Crick equation for calculating the predicted prevalence $\left(\mathrm{p}_{\mathrm{i}}\right)$ for age $\mathrm{e}_{\mathrm{i}}$ :

$$
\mathrm{p}_{\mathrm{i}}=0.0654 /(1+(5776 \times \operatorname{EXP}(-0.1105 \times
$$

The Quigley-Vitale equation for calculating the predicted prevalence $\left(\mathrm{p}_{\mathrm{i}}\right)$ for age $\mathrm{i}_{\mathrm{i}}$ :

$$
\begin{array}{r}
\mathrm{p}_{\mathrm{i}}=\left(1.59 \times 10^{-2}\right)-\left(1.14 \times 10^{-3}\right) \times\left(\mathrm{age}_{\mathrm{i}}-\right. \\
30)+\left(3.39 \times 10^{-5}\right) \times\left(\mathrm{age}_{\mathrm{i}}-30\right)^{2}
\end{array}
$$

For each procedure, the predicted age specific prevalence proportion $\left(\mathrm{p}_{\mathrm{i}}\right)$ was then multiplied by the total number who were of that age $\left(\mathrm{N}_{\mathrm{i}}\right)$ to arrive at the number of POAG cases $\left(\mathrm{C}_{\mathrm{i}}\right)$. The sum of these age specific cases $\left(\sum \mathrm{C}_{\mathrm{i}}\right)$ represented the total number of predicted (expected) POAG cases in the sample, for which the expression $\left(\sum \mathrm{C}_{\mathrm{i}}\right) /\left(\sum \mathrm{N}_{\mathrm{i}}\right)$ gave the overall predicted prevalence. The predicted prevalence proportion was then compared with the actual observed prevalence in the sample.

ESTIMATION OF POPULATION PREVALENCE

Data on the age structure and ethnic mix of the population of England and Wales were obtained by special request from the Office for National Statistics. From these data, the proportion, among those aged 65 or older, belonging to the African-Caribbean ethnic groups were calculated as $35378 / 8044083$ $(0.44 \%)$ and excluded, to arrive at a total projection population of 8008705 people aged 65 or older. These population data were grouped by five " 5 year" age classes, starting from $65-69$, to $80+$.

The prevalence of POAG for each of the five age groups (i), was calculated by substituting the midclass age (for example, 67.5 for the age class 65-69), in the predictive equations, as described above. For the oldest open ended age group, the midclass age was 90 . The overall predicted population prevalence was then computed in the same way as described for the sample.

The same approach was used for estimating the population prevalence from the age specific prevalence data from the North London Eye Study. Here, $\left(\mathrm{p}_{\mathrm{i}}\right)$ was the directly measured prevalence in age group (i) in the NLES sample. The precision of the estimated population prevalence (standard error) was computed using the equation given by Armitage. ${ }^{5}$

The results were presented by 5 year age classes and for the total elderly population of

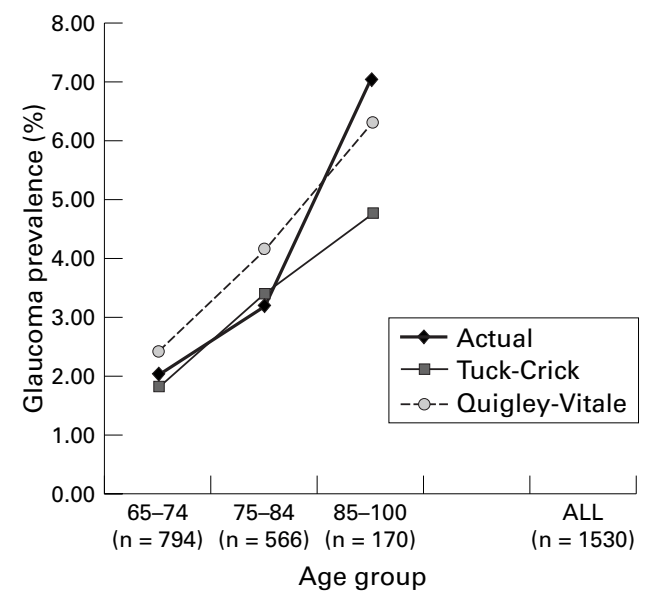

Figure 1 Prevalence of primary open angle glaucoma calculated by the Tuck-Crick and the Quigley-Vitale predictive regression equations, in comparison with the actual prevalence in a random sample of 1530 people aged 65-100, excluding African-Caribbean ethnic groups, from the North London Eye Study.

England and Wales, excluding the small African-Caribbean ethnic group (0.44\%).

For calculations of incidence from age specific prevalence, the simple method proposed by Leske et al $l^{6}$ was used.

\section{Results}

Our directly measured actual prevalence of POAG in the sample of 1530 people (which did not include African-Caribbean ethnic groups) is compared with the prevalence predicted by the application of the QuigleyVitale and the Tuck-Crick equations in Figure 1. The actual directly measured prevalence of POAG in the local sample was $3.01 \%$. The Tuck-Crick estimate was $2.74 \%$ (difference $0.27 \%$ ), and the Quigley-Vitale was $3.50 \%$ (difference $-0.49 \%$ ).

Table 1 outlines the estimated prevalence of POAG in England and Wales, obtained by three methods:

(1) based on the age specific prevalence data from the North London Eye Study

(2) based on the Tuck-Crick equation

(3) based on the Quigley-Vitale equation.

The three methods gave point estimates of $2.85 \% \quad$ (228 526 cases), $2.71 \% \quad$ (217 375 cases), and 3.50\% (280 364 cases) respectively for the prevalence of POAG in the elderly population of England and Wales. The precision of the point estimates could only be reported for method 1 .

Calculation of incidence from the age specific prevalence data reported above gave

Table 1 Prevalence of primary open angle glaucoma in the elderly population of England and Wales (excluding

\begin{tabular}{|c|c|c|c|c|c|c|c|}
\hline \multirow[b]{2}{*}{ Age (years) } & \multirow{2}{*}{$\begin{array}{l}\begin{array}{l}\text { England } \\
\text { and Wales }\end{array} \\
\text { Population }\end{array}$} & \multicolumn{2}{|c|}{ Tuck-Crick equation } & \multicolumn{2}{|c|}{ Quigley-Vitale equation } & \multicolumn{2}{|c|}{$\begin{array}{l}\text { Estimates from the North } \\
\text { London Eye Study }\end{array}$} \\
\hline & & Number & $\%$ & Number & $\%$ & No & $\%(S E)$ \\
\hline $65-69$ & 2471923 & 37343 & 1.5 & 51470 & 2.1 & 39030 & 1.6 \\
\hline $70-74$ & 2005106 & 44971 & 2.2 & 57510 & 2.9 & 48433 & 2.4 \\
\hline $75-79$ & 1658753 & 51593 & 3.1 & 63426 & 3.8 & 40212 & 2.4 \\
\hline $80-84$ & 1111272 & 44462 & 4.0 & 54993 & 4.9 & 47088 & 4.2 \\
\hline $85+$ & 761651 & 39005 & 5.1 & 52965 & 7.0 & 53764 & 7.1 \\
\hline All & 8008705 & 217375 & 2.7 & 280364 & 3.5 & 228526 & $2.9(0.4)$ \\
\hline
\end{tabular}
African-Caribbean ethnic groups), estimated by three methods: application of the Tuck-Crick and the Quigley-Vitale predictive equations, and application of age-specific prevalence data from the North London Eye Study 
the following results. The numbers of new cases of POAG expected (5 year cumulative incidence) in the elderly population of England and Wales were 71146 and 94485 for methods 2 and 3 respectively.

\section{Discussion}

The Tuck-Crick predictive equation produced the closest estimate to the actual directly measured POAG prevalence in the local sample. For the oldest age group, however, the Quigley-Vitale estimate was closer of the two to the actual prevalence. Both predictive equations are based on a number of surveys, incorporating a mixed bag of criteria for diagnosis of POAG. Our local definition of POAG is probably closer to the mix underlying the TuckCrick predictive equation than the mix used in studies upon which the Quigley-Vitale equation is based. Arguments as to which equation is best for predicting the "true" POAG prevalence in general would be baseless since there is no single agreed definition of POAG. We can only assess the goodness of the equations in the context of the NLES criteria as applied to the elderly population in the UK (that is, against our own local POAG definition). In that context, the Tuck-Crick predictive equation is preferred.

The two predictive equations differ both mathematically and in the sources of the prevalence data used to derive them. Quigley and Vitale used linear models with a random effect term representing the prevalence studies, and a fixed effect quadratic term for age, with more weight given to studies that had larger sample sizes. The data sources included two earlier studies from the UK (Bedford 1968, and Melton Mowbray 1985), a study from Sweden (1982), one from Norway (1991), and one from Malta (1989). None of these were used in the derivation of the Tuck-Crick predictive equation, which required POAG to be defined primarily in terms of glaucomatous optic nerve damage. Tuck and Crick fitted a logistic curve to the published prevalence data, giving equal weights to all eight of the source studies. These included a recent large survey from Australia (the Blue Mountains Eye Study 1997) and a smaller Italian survey (the Casteldaccia Eye Study 1995), which were not used by Quigley and Vitale. Six sources of data were used in the derivation of both predictive equations. These were from studies in Baltimore; Beaver Dam; Framingham; Roscommon; Rotterdam; and Ferndale.
For estimating the POAG incidence in the population, the NLES survey data, as for other such data, are limited, so that age specific prevalence cannot be estimated reliably for each year of age. This in turn makes it difficult to estimate 1 year cumulative incidence. The predictive equation methods of Tuck-Crick and Quigley-Vitale, however, allow the calculation of annual (as well as 5 year) incidence and number of new cases for the population. The main utility of the predictive equations is, however, in estimation of prevalence in populations for which there are no sample survey data.

The North London Eye Study indicated a sharper rise in prevalence between the two oldest age groups than predicted by the equations. In view of the expected rise in numbers surviving to beyond 85 years, particularly in the established market economies (Western Europe, North America, Australia, Japan, and New Zealand), reliable estimates of glaucoma prevalence for this oldest stratum will become increasingly important. New survey data, particularly for the very elderly for whom data are sparse, may contribute to the modification of the predictive equations and make them more precise predictors of POAG prevalence in the older population.

Time is now ripe for the key workers in this field to collaborate in sharing data and methods so that projections can be made for large populations, including statements of the levels of uncertainty (for example, 95\% confidence limits) for the prevalence and incidence estimates. These lower and upper limits would strengthen decision making in the provision of ophthalmic services, in that they would give the "minimum" and "maximum" number of cases of POAG expected in the population.

Supported by a grant from North Thames Regional Health Authority R\&D.

1 Quigley HA, Vitale S. Models of open-angle glaucoma prevalence and incidence in the United States. Invest $O p h$ thalmol Vis Sci 1997;38:83-91.

2 Tuck MW, Crick RP. The age distribution of primary open angle glaucoma. Ophthalmic Epidemiol 1998;5:173-83.

3 Reidy A, Minassian DC, Vafidis G, et al. Prevalence of serious eye disease and visual impairment in a north London population: population based, cross sectional study. $B M \mathcal{F}$ 1998;316:1643-6.

4 Jarman B. Underprivileged areas: validation and distribution of scores. BMF 1984;289:1587-92.

5 Armitage P. Statistical methods in medical research. Oxford: Blackwell Scientific Publications, 1980.

6 Leske MC, Ederer F, Podgor M. Estimating incidence from age-specific prevalence in glaucoma. Am f Epidemiol 1981; 113:606-13. 